\title{
Chapter 1 GUSS: Solving Collections of Data Related Models within GAMS
}

\author{
Michael R. Bussieck, Michael C. Ferris, and Timo Lohmann
}

\begin{abstract}
In many applications, optimization of a collection of problems is required where each problem is structurally the same, but in which some or all of the data defining the instance is updated. Such models are easily specified within modern modeling systems, but have often been slow to solve due to the time needed to regenerate the instance, and the inability to use advance solution information (such as basis factorizations) from previous solves as the collection is processed. We describe a new language extension, GUSS, that $g$ athers data from different sources/symbols to define the collection of models (called scenarios), $\boldsymbol{u}$ pdates a base model instance with this scenario data and $s$ olves the updated model instance and $s$ catters the scenario results to symbols in the GAMS database. We demonstrate the utility of this approach in three applications, namely data envelopment analysis, cross validation and stochastic dual dynamic programming. The language extensions are available for general use in all versions of GAMS starting with release 23.7.
\end{abstract}

\subsection{Introduction}

Algebraic modeling systems (such as GAMS, AMPL, AIMMS, etc) are a well established methodology for solving broad classes of optimization problems arising in a wide variety of application domains. These modeling systems take data from a rich collection of sources including databases, spreadsheets, web

Michael R. Bussieck

GAMS Software GmbH, Cologne, e-mail: mbussieck@gams.com

Michael C. Ferris

University of Wisconsin, Madison, Wisconsin e-mail: ferris@cs.wisc.edu

Timo Lohmann

GAMS Development Corp., Washington D.C., e-mail: tlohmann@gams.com 
interfaces and simple text files and are able to process specific instantiations of the often large and complex models using state of the art solution engines.

The purpose of this paper is to detail an extension of the GAMS modeling system that allows collections of models (parameterized exogenously by a set of samples or indices) to be described, instantiated and solved efficiently.

As a specific example, we consider the parametric optimization problem $\mathcal{P}(s)$ defined by:

$$
\min _{x \in X(s)} f(x ; s) \text { s.t. } g(x ; s) \leq 0
$$

where $s \in S=\{1, \ldots, K\}$. Note that each scenario $s$ represents a different problem for which the optimization variable is $x$. The form of the constraint set as given above is simply for concreteness; equality constraints, range and bound constraints are trivial extensions of the above framework. Clearly the problems $\mathcal{P}(s)$ are interlinked and we intend to show how such problems can be easily specified within GAMS and detail one type of algorithmic extension that can exploit the nature of the linkage. Other extensions of GAMS allow solves to be executed in parallel or using grid computing resources [6].

The paper is organized as follows. In Section 1.2 we outline the motivation and design of the system for describing and solving $\mathcal{P}(s)$ and give some overview of the available options. Section 1.3 gives three examples of the use of this methodology to the problems of data envelopment analysis (DEA - Section 1.3.1), cross validation for problems in machine learning (Section 1.3.2) and finally to stochastic dual dynamic programming (SDDP - Section 1.3.3). Note that in our description we will use the terms indexed, parameterized and scenario somewhat interchangeably. Furthermore we assume that the reader has basic knowledge about the modeling language GAMS.

\subsection{Design Methodology}

One of the most important functions of GAMS is to build a model instance from the collection of equations (i.e. an optimization model defined by the GAMS keyword MODEL) and corresponding data (consisting of the content of GAMS (sub)sets and parameters). Such a model instance is constructed or generated when the GAMS execution system executes a SOLVE statement. The generated model instance is passed to a solver which searches for a solution of this model instance and returns status information, statistics, and a (primal and dual) solution of the model instance. After the solver terminates, GAMS brings back the solution into the GAMS database, i.e. it updates the level (.L) and marginal (.M) fields of variable and equation symbols used in the model instance. Hence, the SOLVE statement can be interpreted as a com-

plex operator against the GAMS database. The model instance generated by a SOLVE statement only lives during the execution of this one statement, and hence has no representation within the GAMS language. Moreover, its struc- 
ture does fit the relational data model of GAMS. A model instance consists of vectors of bounds and right hand sides, a sparse matrix representation of the Jacobian, a representation of the non-linear expressions that allow the efficient calculation of gradient vectors and Hessian matrices and so on.

This paper is concerned with solving collections of models that have similar structure but modified data. As an example, consider a linear program of the form:

$$
\min c^{T} x \text { s.t. } A x \geq b, \ell \leq x \leq u .
$$

The data in this problem is $(A, b, c, \ell, u)$. Omitting some details, the following code could be used within GAMS to solve a collection of such linear programs in which each member of the collection has a different $A$ matrix and lower bound $\ell$ :

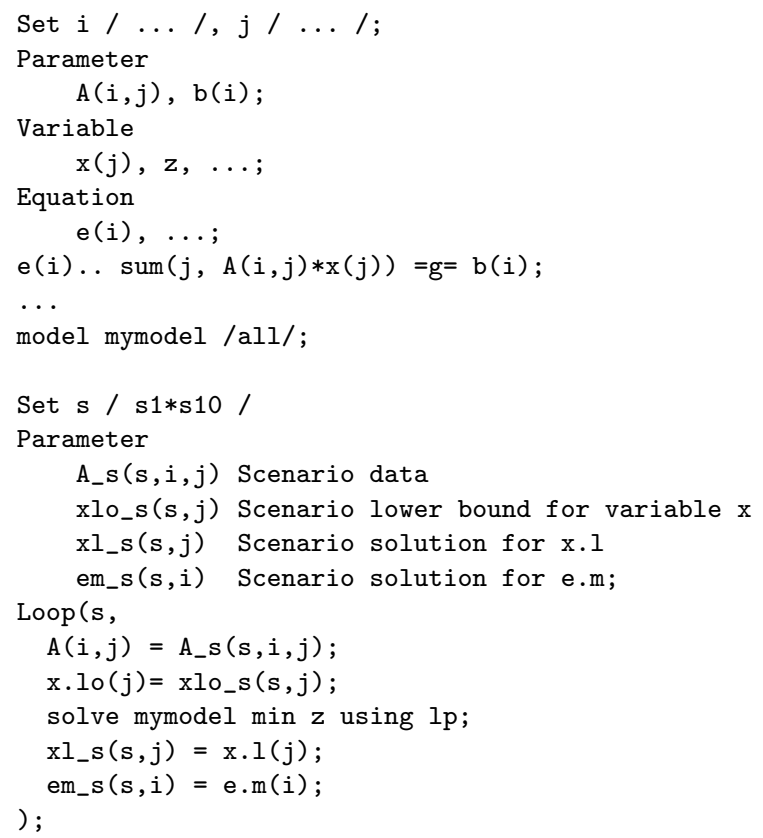

Summarizing, we solve one particular model (mymodel) in a loop over s with an unchanged model rim (i.e. the same individual variables and equations) but with different model data and different bounds for the variables. The change in model data for a subsequent solve statement does not depend on the previous model solutions in the loop.

The purpose of this new Gather-Update-Solve-Scatter manager or short GUSS is to provide syntax at the GAMS modeling level that makes an instance of a problem and allows the modeler limited access to treat that instance as an object, and to update portions of it iteratively. Specifically, we provide syntax that gives a list of data changes to an instance, and allows these changes to be applied sequentially to the instance (which is then solved 
without returning to GAMS). Thus, we can simulate a limited set of actions to be applied to the model instance object and retrieve portions of the solution of these changed instances back in the modeling environment.

Such changes can be done to any model type in GAMS, including nonlinear problems and mixed integer models. However, the only changes we allow are to named parameters appearing in the equations and lower and upper bounds used in the model definition.

Thus, in the above example GUSS allows us to replace lines 18-24 by

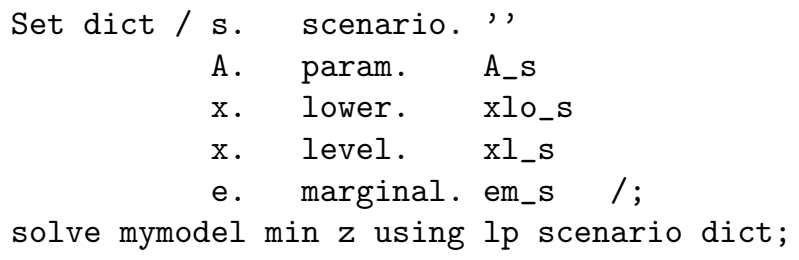

The three dimensional set dict (you can freely choose the name of this symbol) contains mapping information between symbols in the model (in the first position) and symbols that supply required update data or store solution information (in the third position), and the type of update/storing (in the second position). An exception to this rule is the tuple with label scenario in the second position. This tuple determines the symbol (in the first position) that is used as the scenario index. This scenario symbol can be a multidimensional set. A tuple in this set represents a single scenario. The remaining tuples in the set dict can be grouped into input and output tuples. Input tuples determine the modifications of the model instance prior to solving, while output tuples determine which part of the solution gets saved away. The following keywords can be used in the second position of the set dict:

Input:

param: Supplies scenario data for a parameter used in the model

lower: Supplies scenario lower bounds for a variable

upper: Supplies scenario upper bounds for a variable

fixed: Supplies scenario fixed bounds for a variable

Output:

level: Stores the levels of a scenario solution of variable or equation marginal: Stores the marginals of a scenario solution of variable

or equation

Sets in the model cannot be updated. GUSS works as follows: GAMS generates the model instance for the original data. As with regular SOLVE statements, all the model data (e.g. parameter A) needs to be defined at this time. The model instance with the original data is also called the base case. The solution of the base case is reported back to GAMS in the regular way and is accessible via the regular .L and .M fields after the SOLVE statement. 
After solving the base case, the update data for the first scenario is applied to the model. The tuples with lower, upper, fixed update the bounds of the variables, whereas the tuples with param update the parameters in the model. The scenario index $\mathrm{k}$ needs to be the first index in the parameters mapped in the set dict. The update of the model parameters goes far beyond updating the coefficients of the constraint matrix/objective function or the right hand side of an equation as one can do with some other systems. GAMS stores with the model instance all the necessary expressions of the constraints, so the change in the constraint matrix coefficient is the result of an expression evaluation. For example, consider a term in the calculation of the cost for shipping a variable amount of goods $x(i, j)$ between cities $i$ and $j$. The expression for shipping cost is $d(i, j) * f * x(i, j)$, i.e. the distance between the cities times a freight rate $f$ times the variable amount of goods. In order to find out the sensitivity of the solution with respect to the freight rate $f$, one can solve the same model with different values for $f$. In a matrix representation of the model one would need to calculate the coefficient of $x(i, j)$ which is $d(i, j) * f$, but with GUSS it is sufficient to supply different values for $f$ that potentially result in many modified coefficient on the matrix level. The evaluation of the shipping cost term and the communication of the resulting matrix coefficient to the solver are done reliably behind the scenes by GUSS.

After the variable bound and the model parameter updates have been applied and the resulting updates to the model instance data structures (e.g. constraint matrix) has been determined, the modified model instance is passed to the solver. Some solvers (e.g. Cplex, Gurobi, and Xpress) allow modifying a model instance. So in such a case, GUSS only communicates the changes from the previous model instance to the solver. This not only reduces the amount of data communicated to the solver, but also, in the case of an LP model, allows the solver to restart from an advanced basis and its factorization. In the case of an NLP model, this provides initial values. After the solver determines the solution of a model instance, GUSS stores the part of the solution requested by the output tuples of dict to some GAMS parameters and continues with the next scenario.

\subsubsection{GUSS Options}

The execution of GUSS can be parameterized using some options. Options are not passed through a solver option file but via another tuple in the dict set. The keyword in the second position of this tuple is opt. A one dimensional parameter is expected in the first position (or the label ' '). This parameter may contain some of the following labels with values: 
OptfileInit: Option file number for the first solve

Optfile: Option file number for subsequent solves

LogOption: Determines amount of log output:

0 - Moderate log (default)

1 - Minimal log

2 - Detailed log

SkipBaseCase: Switch for solving the base case (0 solves the base case)

UpdateType: Scenario update mechanism:

0 - Set everything to zero and apply changes (default)

1 - Reestablish base case and apply changes

2 - Build on top of last scenario and apply changes

RestartType: Determines restart point for the scenarios

0 - Restart from last solution (default)

1 - Restart from solution of base case

2 - Restart from input point

For the example model above the UpdateType setting would mean:

$$
\begin{array}{rr}
\text { UpdateType=0: } & \operatorname{loop}\left(s, A(i, j)=A_{-} s(s, i, j)\right) \\
\text { UpdateType=1: } & \operatorname{loop}\left(s, A(i, j)=A_{-} b a s e(i, j) ;\right. \\
\left.A(i, j) \quad \$=A \_s(s, i, j)\right) & \text { UpdateType=2: } \quad \operatorname{loop}\left(s, A(i, j) \quad \$=A \_s(s, i, j)\right)
\end{array}
$$

The option SkipBaseCase=1 allows to skip the base case. This means only the scenarios are solved and there is no solution reported back to GAMS in the traditional way. The third position in the opt-tuple can contain a parameter for storing the scenario solution status information, e.g. model and solve status, or needs to have the label ' '. The labels to store solution status information must be known to GAMS, so one needs to declare a set with such labels. The following solution status labels can be reported:

$\begin{array}{llllll}\text { domusd } & \text { iterusd } & \text { objest } & \text { nodusd } & \text { modelstat } & \text { numnopt } \\ \text { numinfes } & \text { objval } & \text { rescalc } & \text { resderiv } & \text { resin } & \text { resout } \\ \text { resusd } & \text { robj } & \text { solvestat } & \text { suminfes } & & \end{array}$

The following example shows how to use some of the GUSS options and the use of a parameter to store some solution status information:

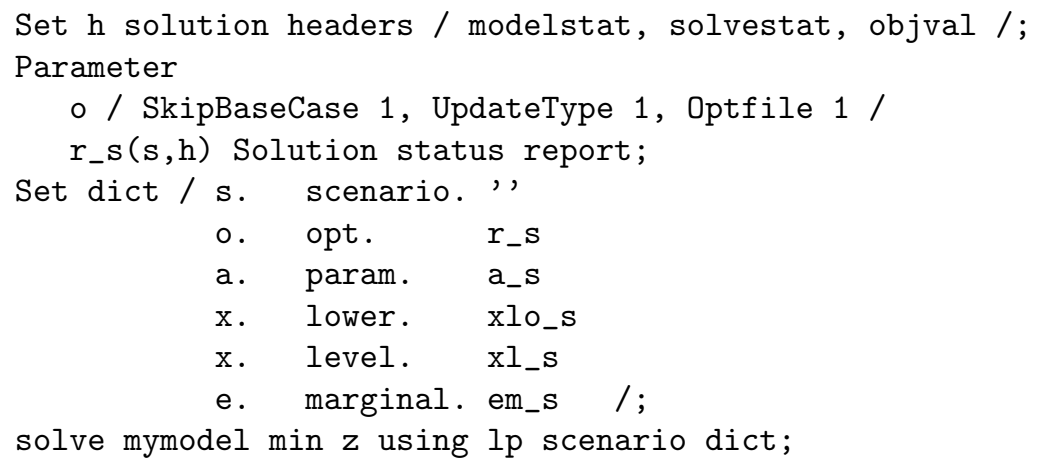




\subsubsection{Implementation Details}

This section describes some technical details that may provide useful insight in case of unexpected behavior.

GUSS changes all model parameters mentioned in the dict set to variables. So a linear model can produce some non-linear instructions (e.g. $d(i, j) * f * x(i, j)$ becomes a non-linear expression since $f$ becomes a variable in the model instance given to GUSS). This also explains why some models compile without complaint, but if the model is used in the context of GUSS, the compile time check of the model will fail because a parameter that is turned into a variable cannot be used that way any more. For example, suppose the model contains a constraint e $(i) \ldots \operatorname{sum}(j \$ A(i, j), \ldots)$. If $A(i, j)$ is a parameter in the regular model, the compiler will not complain, but if $\mathrm{A}$ becomes a parameter that shows up in the first position of a param tuple in the dict set, the GAMS compiler will turn A into a variable and complain that an endogenous variable cannot be used in a $\$$-condition.

The sparsity pattern of a model can be greatly effected by GUSS. In a regular model instance GAMS will only generate and pass on non-zero matrix elements of a constraint $e(i) \ldots \operatorname{sum}(j, A(i, j) * x(j)) \ldots$, so the sparsity of A determines the sparsity of the generated model instance. GUSS allows to use this constraint with different values for A hence GUSS cannot exclude any of the pairs $(i, j)$ and generate a dense matrix. The user can enforce some sparsity by explicitly restricting the $(i, j)$ pairs: $e(i) \ldots \operatorname{sum}(i j(i, j), A(i, j) * x(j)) \ldots$

The actual change of the GAMS language required for the implementation of GUSS is minimal. The only true change is the extension of the SOLVE statement with the term SCENARIO dict. Existing language elements have been used to store symbol mapping information, options, and model result statistics. Some parts of the GUSS presentation look somewhat unnatural, e.g. since dict is a three dimensional set the specification the scenario set using keyword scenario requires a third dummy label ',. However, this approach gives maximum flexibility for future extension, allows reliable consistency checks at compile and execution time, and allows to delay the commitment for significant and permanent syntax changes of a developing method to handle model instances at a GAMS language level.

\subsection{Examples}

In this section we discuss three examples that benefit from GUSS. Data envelopment analysis models are discussed in section 1.3.1 and a discussion about cross validation models can be found in section 1.3.2. These example describe in detail the steps from a traditional GAMS implementation to a GUSS based

model. In section 1.3.3 we present the use of GUSS in an implementation 
of the stochastic dual dynamic program. As many other decomposition algorithms SDDP requires the solution of many closely related mathematical optimization problems. We discuss in detail the savings in running time when using GUSS compared to a traditional GAMS implementation and even an implementation based on a native solver interface.

\subsubsection{Data Envelopment Analysis}

Data Envelopment Analysis (DEA) models have been used extensively in the literature to study a wide variety of applications [9, 10, 13, 15, 18, 22, 24]. The basic (CCR) DEA model is a collection of models indexed by $\mathrm{k}$ and defined by

$$
\begin{array}{lll}
\max _{u, v} & u^{T} Y_{\cdot k} \quad \text { (indexed objective) } \\
\text { s.t. } & v^{T} X \cdot k=1 \quad \text { (indexed normalizing constraint) } \\
& u^{T} Y \leq v^{T} X \\
& u, v \geq 0
\end{array}
$$

where $X, Y$ are data matrices. The complete GAMS models discussed in the section including the data is available from the GAMS/DEA web page at http://www.gams. com/contrib/gamsdea/dea.htm.

Without GUSS, a model would be defined and solved in a loop over k, requiring the model to be generated multiple times with different instances for each value of $\mathrm{k}$ as shown below.

\subsubsection{Standard loop version of primal DEA model}

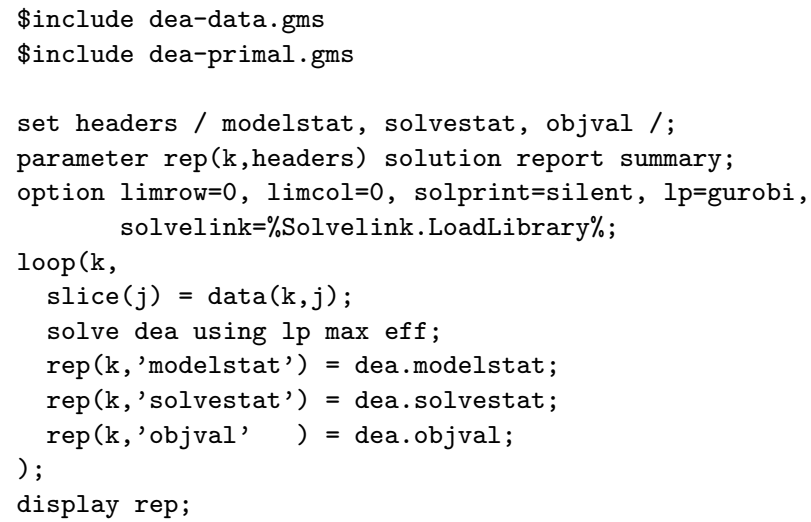


In this setting we loop over the set $\mathrm{k}$ and change the data in the objective function and the first constraint of the model explicitly before each solve. We only output a minimal summary of the solution.

GUSS is an alternative (and more efficient) way to define the individual programs and pass them to any underlying GAMS solver. In this way, individual programs are not regenerated, but are instead defined as data modifications of each other. This reduces overall model generation time. Further, previous solutions can be used as starting points in later solves to speed up overall processing time. The specific GAMS code to achieve this is shown below.

\subsubsection{GUSS version of primal DEA model}

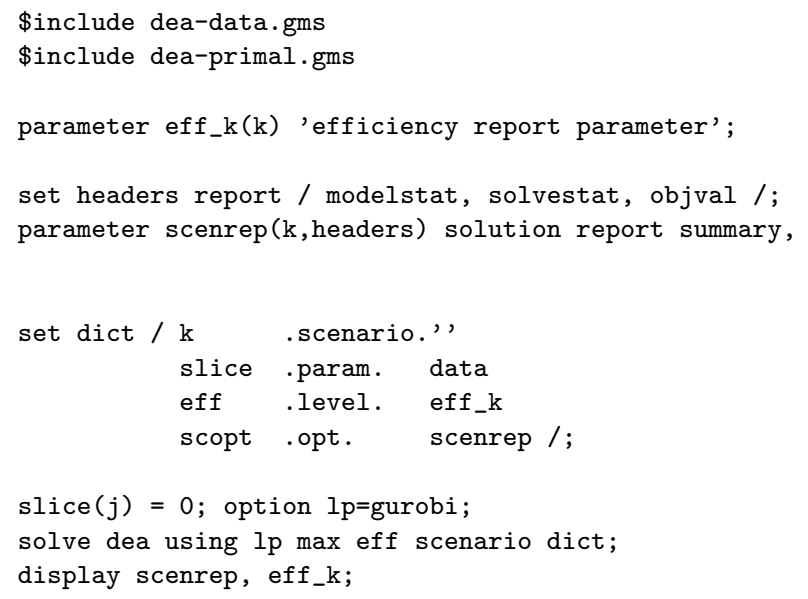

In the GUSS version we indicate the collection of models to be solved using the set dict defined on lines 10-13. The solve statement on line 16 includes an extra keyword scenario that points to this set. The contents of dict are directives to GUSS. The first tuple of dict determines the set to be used for the scenario (collection) index, in this case $k$. The second tuple of dict states that in each scenario $k$, the parameter slice is instantiated using a slice of the parameter data. Essentially, this corresponds to the GAMS statement:

$\operatorname{slice}(j)=\operatorname{data}(k, j)$

Note the scenario index $\mathrm{k}$ must appear as the first index of the parameter data. The third tuple of dict allows the modeler to collect information from each solve and store it into a GAMS parameter. Essentially, the third element of dict corresponds to the GAMS statement:

eff_k $(k)=\operatorname{eff} .1$ 
that gets executed immediately after the solve of scenario k. GUSS options (scopt) and a parameter to store model statistics (scenrep) are given in the last tuple of dict indicated by the keyword opt.

More complex scenario models can also be formulated using GUSS, including multiple equations being updated. This is shown by the dual of the basic DEA model, given by

$$
\begin{array}{lll}
\min _{z, \lambda} & & \text { (objective) } \\
\mathrm{s.t.} & X \lambda \leq z X_{\cdot k} & \text { (indexed constraint) } \\
& Y \lambda \geq Y_{\cdot k} \quad \text { (indexed constraint) } \\
& \lambda \geq 0 &
\end{array}
$$

The original GAMS formulation using standard loops is explicitly given below:

\subsubsection{Standard loop version of dual DEA model}

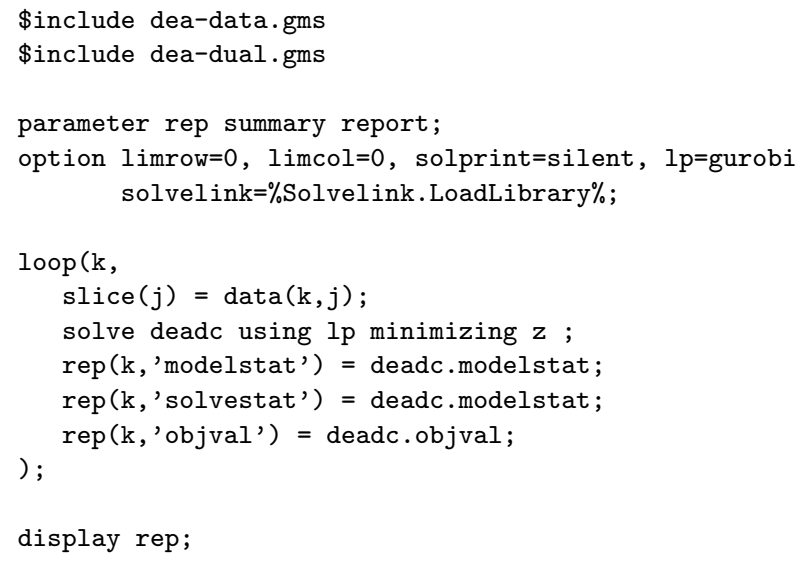

The dual (CRS) DEA model formulated using GUSS is a simple modification, namely:

\subsubsection{GUSS version of dual DEA model}

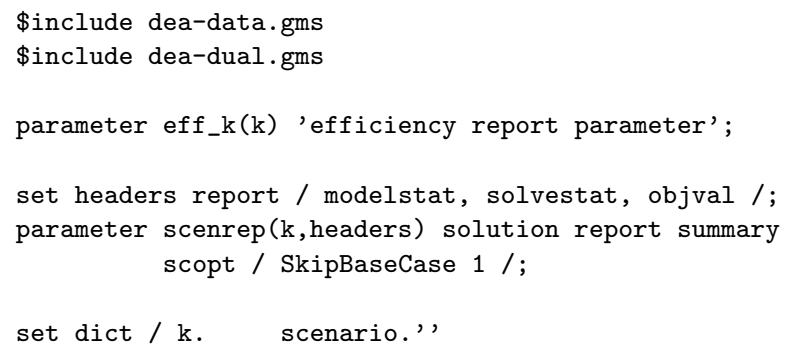




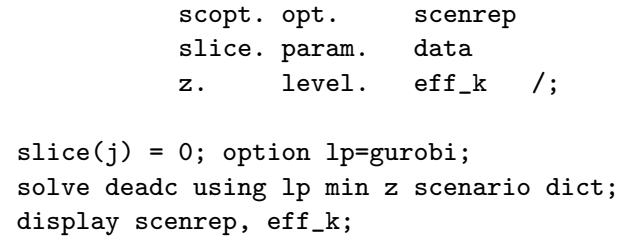

Because the base model is not solved (due to option SkipBaseCase 1), no solution is reported back to GAMS in the traditional way. Solutions for all of the programs can be collected into GAMS parameters as shown above for the eff_k parameter.

The aforementioned model is not the only DEA model that exists. Other DEA models that address application issues have been developed and used in practice. Some of these models are simple modifications of the CCR model; other vary more. With the variety of models available, each addressing different needs, GAMS is an important tool to facilitate the definition of general DEA models; GUSS enables fast solution.

Further extensions of these models $[1,2,3,8,23]$ to formulations with weighted outputs or variable returns to scale are easy to formulate with GUSS. One such extended model is also given on the GAMS/DEA web page. It implements the following primal dual pair that incorporates variable returns to scale (VRS) and additive modeling:

$$
\begin{array}{ll}
\max _{u, v} & u^{T} Y_{\cdot k}-\mu \\
\text { s.t. } & v^{T} X_{\cdot k}=100 \\
& u^{T} Y \leq v^{T} X+\mu e \\
& u \geq u_{l o}, v \geq v_{l o}
\end{array}
$$

and

$$
\begin{array}{ll}
\min _{z, \lambda, s, t} & 100 z-u_{l o}^{T} s-v_{l o}^{T} t \\
\text { s.t. } & X \lambda+s=z X_{\cdot k} \\
& Y \lambda-t=Y_{\cdot k} \\
& e^{T} \lambda=1 \\
& \lambda, s, t \geq 0
\end{array}
$$

\subsubsection{Cross Validation for Support Vector Machines}

Cross validation $[16,17,21,12]$ is a statistical/machine learning technique that aims to evaluate the generalizability of a classifier (or other decision) process. It does this by setting aside a portion of the data for testing, and uses the remaining data entries to produce the classifier. The testing data is subsequently used to evaluate how well the classifier works. Cross validation performs this whole process a number of times in order to estimate the true power of the classifier. 
Ten-fold cross validation is a special case, where the original data is split into ten pieces, and cross validation is performed using each of these ten pieces as the testing set. Thus, the training process is performed ten times, each of which uses the data obtained by deleting the testing set from the whole dataset. We show below how to carry this out using GUSS.

The following example compares the two formulations for a featureselection model under cross-validation. The complete model and data files are available from the GAMS/Cross Validation web page at http://www. gams.com/contrib/gamsdea/dea-cv.htm.

Original GAMS formulation:

\subsubsection{Cross validation model}

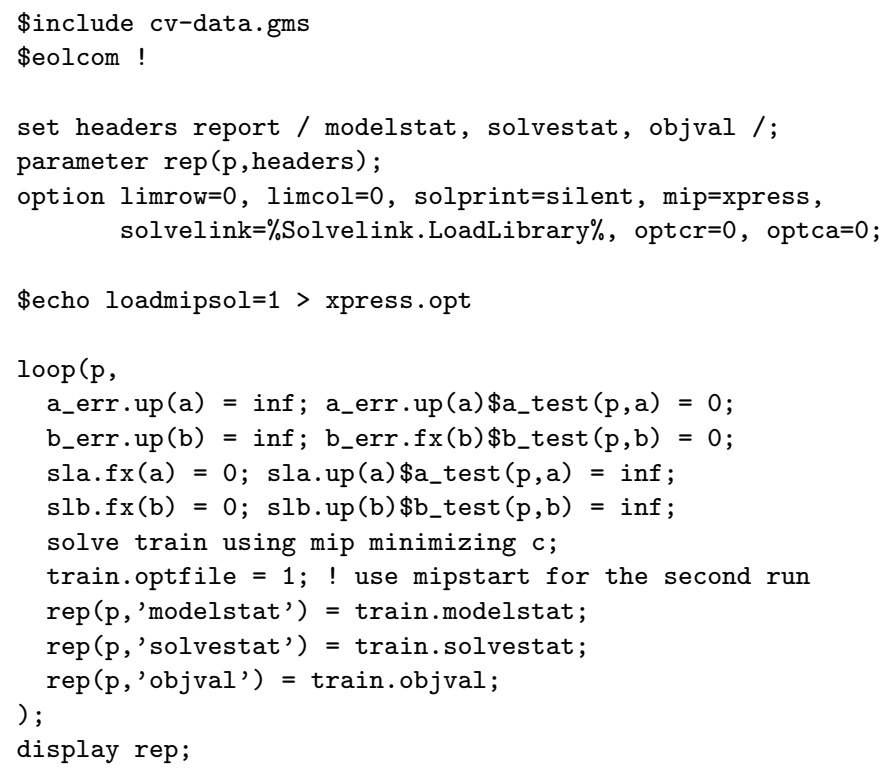

The batinclude file gentestset.inc gives instructions for generating the testing sets. It produces a_test and b_test that detail which equations are left out on solve $\mathrm{p}$.

The actual model is set up to include all the data points in the equations a_def and b_def. To delete the equations that correspond to the test set, we introduce nonnegative slack variables into all the equations. We then set the upper bounds of the slack variables to zero in equations corresponding to the training set, and to infinity in equations corresponding to the testing set. At the same time we fix the error measures $a_{-}$err and $b_{-}$err belonging to the testing set by setting their upper bounds to zero. Thus the testing set equations are always satisfiable by choice of the slack variables alone - essentially they are discarded from the model as required. An alternative formulation 
could "include" the data equations that you need in each scenario, but the update from one scenario to the next in the defining data is much larger.

Cross validation formulated with GUSS: This model essentially mimics what the standard model does, but the implementation of the solver loop behind the scenes is much more efficient.

\subsubsection{Cross validation using GUSS}

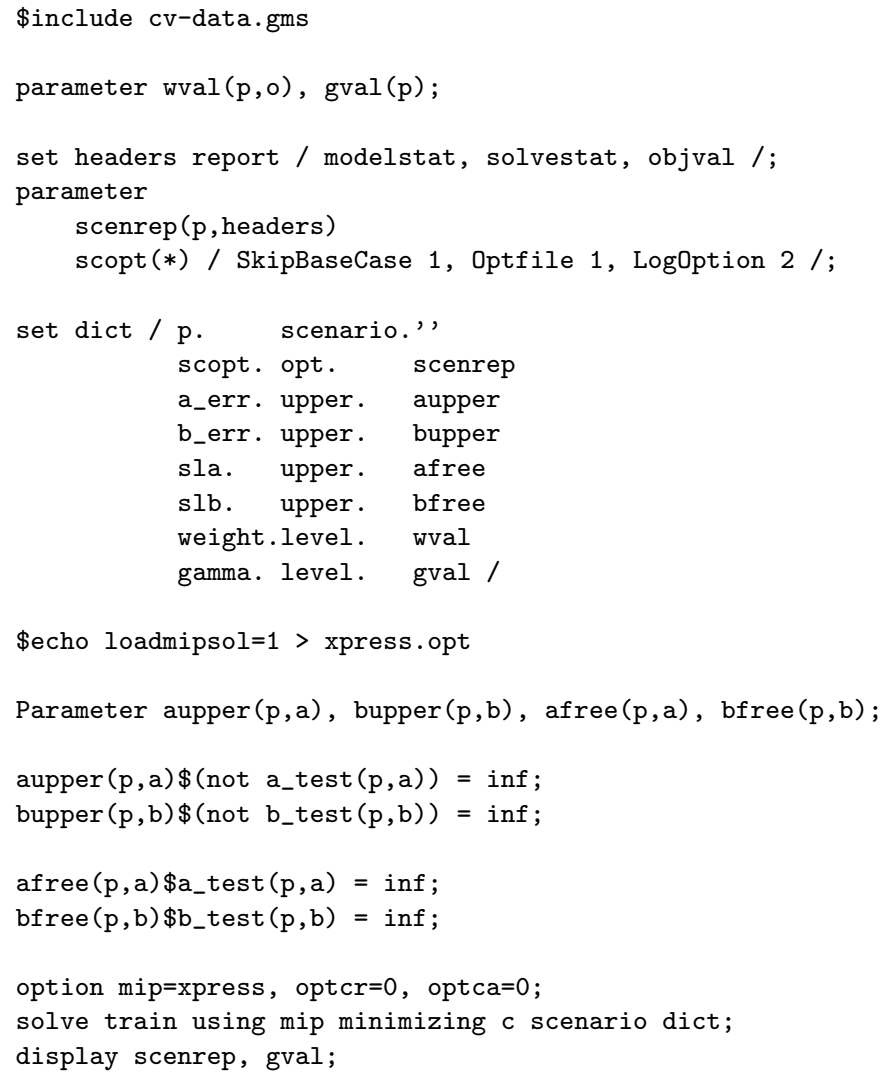

The key observations on this implementation are as follows. Firstly, parameters aupper, bupper, afree and bfree are used to set the bounds on the error and slack variables in the testing set equations respectively. The setting of the upper bounds are governed by the syntax shown in the controlling set dict. Furthermore, the output of the classifier (weight, gamma) for each fold of the cross validation uses the dict' set to place results into the parameters wval and gval respectively. Finally, the GUSS options are used to guarantee that the subsequent solves are instructed to process solver options (Optfile 1) which instruct the solver to use the previous solution to start the branch-and-cut process (loadmipsol=1). 


\section{Quadratic Programs}

GUSS is not limited to linear programs, but can be used more generally. The following example illustrates the use of GUSS for quadratic programs. In this example, a support vector machine is used to determine a linear classifier that separates data into two categories. We use the following model:

$$
\begin{array}{ll}
\min _{w, g, z} & \frac{1}{2}\|w\|_{2}^{2}+C \sum_{i} z_{i} \\
\text { s.t. } & D(A w-g)+z \geq 1 \\
& z \geq 0
\end{array}
$$

Here, $A$ is a matrix containing the training data (patients by features) and $D$ is a diagonal matrix with values +1 or -1 (each denoting one of the two classes). $C$ is a parameter weighting the importance of maximizing the margin between the classes $\left(\frac{2}{\|w\|_{2}}\right)$ versus minimizing the misclassification error $(z)$. The solution $w$ and $g$ are used to define a separating hyperplane $\left\{x \mid w^{T} x=g\right\}$ to classify (unseen) data points.

As given, the standard linear support vector machine is not a slice model per se. It becomes a slice model under cross validation training, where it is solved multiple times on different pieces of data. In this case, only the data $A$ and $D$ vary between solves, appropriately fitting the definition of a slice model.

The data for this example comes from the Wisconsin Diagnosis Breast Cancer Database, and is available at http://www.cs.wisc.edu/ olvi/uwmp/ cancer.html. The data was converted to the GAMS file wdbc.gms, which defines $A$ and $D$ and is also available from the GAMS/Cross Validation web page.

\subsubsection{The GUSS formulation for quadratic SVM}

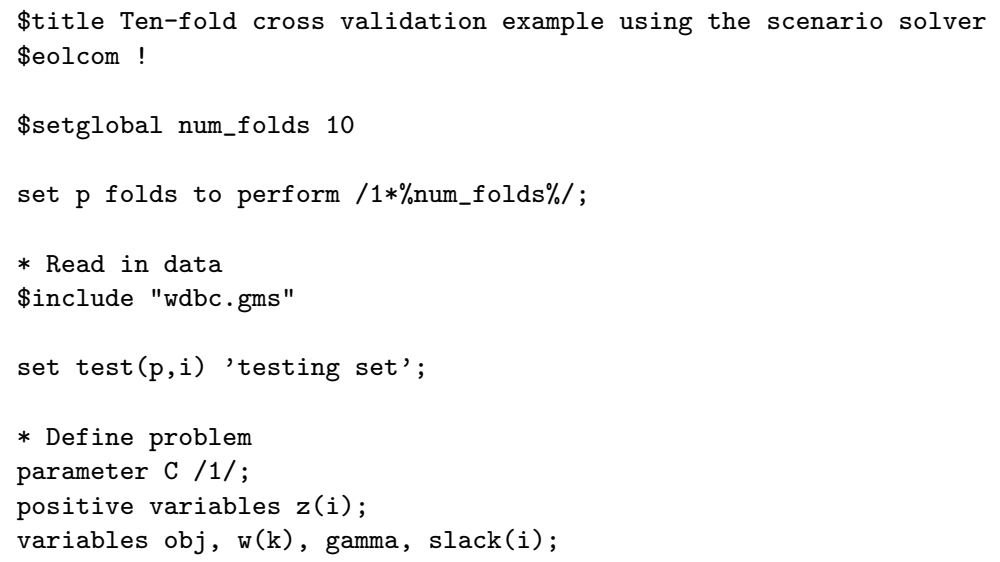




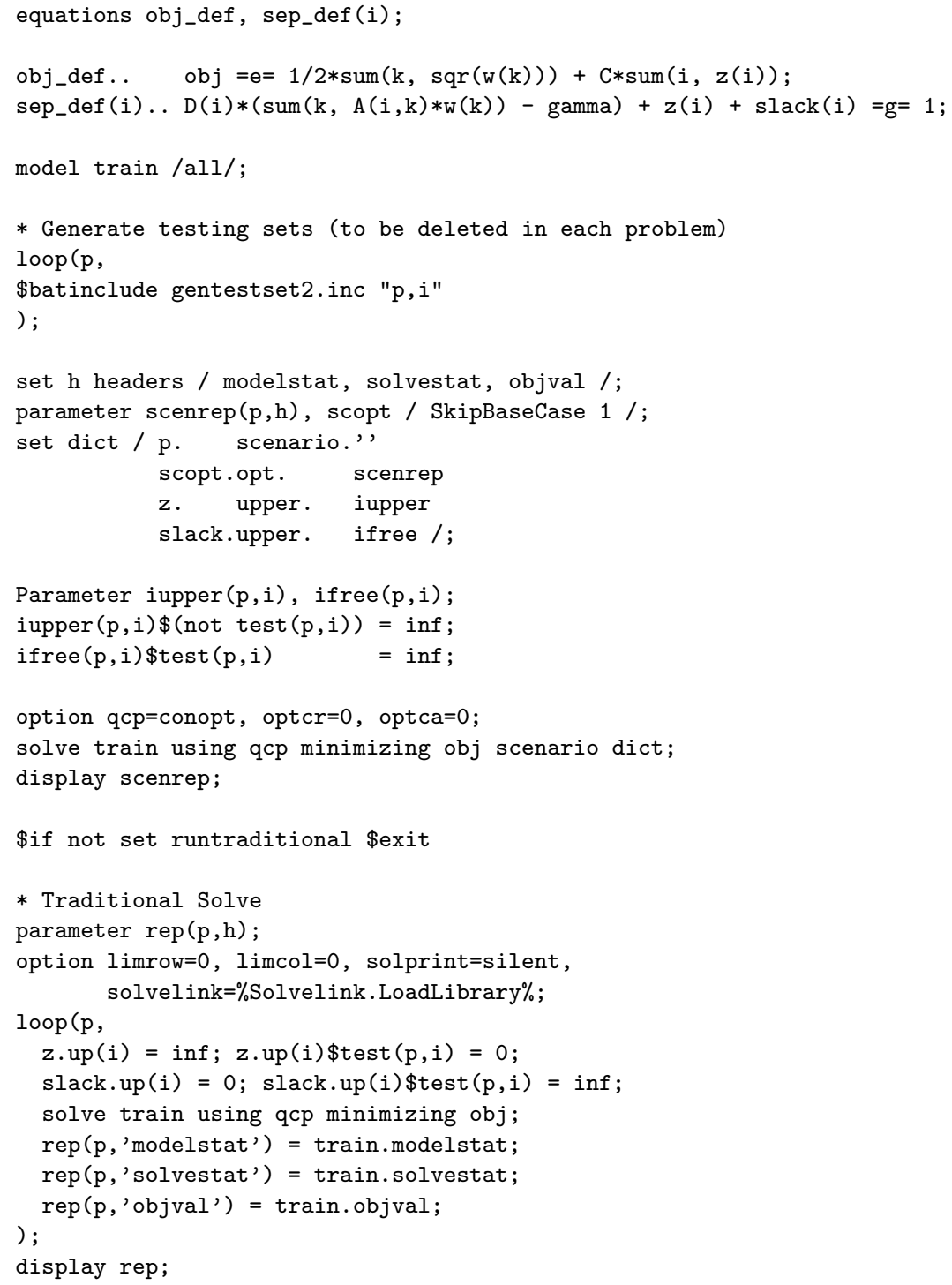

The variable values for weight and gamma could be saved for later testing using the same method as detailed above for the linear case.

The batinclude file gentestset2.inc is very similar to gentestset.inc from the earlier cross-validation examples. In gentestset 2 . inc, though, only one set is being dealt with rather than two. 


\subsubsection{SDDP}

In the last two sections we did not quantify the performance improvements achieved by GUSS. In this section we explore the use of GUSS in a decomposition algorithm applied to a large scale model. We discuss in detail the running times of a traditional GAMS implementation and a GUSS version of the implementation. We also compare the running time of an implementation of the algorithm using the ILOG Concert Technology interface to the Cplex solver.

The Stochastic Dual Dynamic Programming (SDDP) algorithm [19, 20, 25] for solving multi-stochastic linear programs uses, similar to the well known Benders decomposition [4], the concept of a future cost function (FCF). The algorithm works with an underestimating approximation of this FCF by iteratively adding supporting hyperplanes (Benders cuts) and therefore improving the approximation. Let us consider the following multi-stage stochastic linear program [5]

$$
\begin{array}{ll}
\min & c_{1} x_{1}+E\left[\min c_{2}\left(\xi_{2}\right) x_{2}\left(\xi_{2}\right)+\cdots+E\left[\min c_{H}\left(\xi_{H}\right) x_{H}\left(\xi_{H}\right)\right] \cdots\right] \\
\text { s.t. } & W_{1} x_{1}=h_{1} \\
& T_{1}\left(\xi_{2}\right) x_{1}+W_{2}\left(\xi_{2}\right) x_{2}\left(\xi_{2}\right)=h_{2}\left(\xi_{2}\right) \\
& \vdots \\
& T_{H-1}\left(\xi_{H}\right) x_{H-1}\left(\xi_{H-1}\right)+W_{H}\left(\xi_{H}\right) x_{H}\left(\xi_{H}\right)=h_{H}\left(\xi_{H}\right) \\
& x_{1} \geq 0, x_{t}\left(\xi_{t}\right) \geq 0, t=2, \ldots, H
\end{array}
$$

where $\xi_{t}$ are random variables. The SDDP algorithm requires a Markovian structure of the coefficient matrix, meaning that a stage only depends on the previous stage. Furthermore the random variables must be stage-wise independent and must follow a discrete distribution. This means $\xi_{t}$ can be described as $\xi_{t}=\left(\xi_{1 t}, \ldots, \xi_{I t}\right)$ for a discrete distribution of $I$ realizations with respective probability $p_{i}$.

The SDDP algorithm decomposes the stochastic linear problem into $H$ subproblems of the form

$$
\begin{array}{ll}
\min & c_{t} x_{t}+\hat{\alpha}_{t+1} \\
\text { s.t. } & W_{t} x_{t} \geq h_{t}-T_{t-1} x_{t-1}^{*}, \\
& \hat{\alpha}_{t+1}+\pi_{t+1}^{j} T_{t} x_{t} \geq \delta_{t}^{j}, j=1, \ldots, J, \quad\left(S U B\left(t, \xi_{i t}, x_{t-1}^{*}\right)\right) \\
& x_{t} \geq 0,
\end{array}
$$

where $\hat{\alpha}_{t+1}$ is represented by free scalar variables. For reason of convenience we omit the random variables in the description. In order to be able to solve a subproblem the previous stage decision variable $x_{t-1}^{*}$ must be fixed and therefore goes to the right hand side. The set $j=1, \ldots, J$ denotes the added hyperplanes to the subproblem, serving as an approximation of the FCF. Throughout the algorithm these kind of subproblems, with different parameters and variable fixings, are the only problems solved. In order to avoid 
generating and solving them one at a time GUSS allows solving them in certain batches, generating the submodel once for each batch. The details of this process will be shown later in this section.

\subsubsection{Building of cuts}

Each iteration of the SDDP algorithm consists of two phases: a backward recursion and a forward simulation. In the backward recursion supporting hyperplanes of the form

$$
\hat{\alpha}_{t+1}+\pi_{t+1}^{j} T_{t} x_{t} \geq \delta_{t}^{j},
$$

are added to the subproblems in order to improve the approximate FCF. Suppose we are in iteration $j$ and stage $t+1$ of the backward recursion of the algorithm and have solved the subproblem $S U B\left(t+1, \xi_{i, t+1}, x_{t-1}^{*}\right)$ for all $i=1, \ldots, I$ and dual multipliers $\pi_{i, t+1}$ on all constraints with variables of stage

$t$ are stored. In particular this means we may have dual multipliers $\lambda_{i, k, t+1}^{j}$ belonging to cuts that have been added in earlier iterations. We are now moving to the previous stage $t$ and want to build $C U T_{t}^{j}$. For the coefficient of the cut the sum $\pi_{t+1}^{j}=\sum_{i=1}^{I} p_{i} \pi_{i, t+1}$ is calculated. Calculating $\lambda_{k, t+1}^{j}$ is done in the same way. The cut right hand side $\delta_{t}^{j}$ is then calculated as follows

$$
\delta_{t}^{j}= \begin{cases}\sum_{i=1}^{I} p_{i} \pi_{i, t+1}^{j} h_{i, t+1}, & t=H-1 \\ \sum_{i=1}^{I} p_{i} \pi_{i, t+1}^{j} h_{i, t+1}+\sum_{k=1}^{j} \lambda_{k, t+1}^{j} \delta_{t+1}^{k}, & t=1, \ldots, H-2 .\end{cases}
$$

A lower bound is computed by solving the first-stage subproblem with cuts. In the forward simulation the approximate FCF is used to construct a feasible solution of the problem, resulting in an upper bound to the optimal solution value. While going forward we sample one realization out of the set $\left\{\xi_{1 t}, \ldots, \xi_{I t}\right\}$ and solve the respective subproblem. Note that the only cuts described in this section are optimality cuts. Usually a second type of cut, feasibility cuts, are used for a Benders decomposition. By adding slack variables we made sure that these are not needed for our model.

\subsubsection{Algorithm in pseudo-code}

In the actual algorithm both the backward and forward part is passed through with multiple solutions in one iteration. These solutions are called trial solutions and they are important for several reasons. In the backward recursion this leads to one additional cut per trial solution, which results in a better approximation of the FCF. In the forward simulation the trial solutions help 
to get a more reasonable estimate of the upper bound. The algorithm in pseudo-code reads as follows

1: while convergence is not reached do

for $t=H, \ldots, 2$ (Backward recursion) do

for each trial solution $x_{t-1}^{*}$ do

for each realization $\xi_{i t}$ of the random variable do Solve $S U B\left(t, \xi_{i t}, x_{t-1}^{*}\right)$ and calculate dual multipliers of the constraints.

6:

GUSS allows us to rewrite the various SOLVE statements in GAMS in the inner loop of the backward recursion (lines 3-7) into one SOLVE statement. In specific, we write all possible combinations of trial solutions and realizations into the scenario dict. This results in one SOLVE statement per stage and each of these SOLVE statements will solve (\#trials $\times$ \#realizations) many models without regenerating them. In the forward simulation we can rewrite the SOLVE statements in GAMS in the inner loop (lines 11-12). This again results in one SOLVE statement per stage instead of having \#trials many SOLVE statements.

\subsubsection{Results}

The SDDP algorithm has been implemented for a stochastic linear program motivated by Vattenfall Energy Trading, a branch of the Swedish power company Vattenfall. The objective of the model is to minimize the power generation costs and ultimately to forecast power prices of the market. Power can be generated by an aggregated hydro power plant, a coal plant, or a nuclear 
plant. Using hydro power has no costs, but there is a limited amount of water in the reservoir and limited inflows of water into the reservoir over time. In each time period, water can be either used for power generation, saved, or spilled. The model has a granularity of hours and is set up for one year, which results in 8736 time periods. Stochastic information is revealed every week, resulting in 52 stages. A one-stage sub-model therefore consists of 168 hours. In order to compare different implementations of the algorithm, we recorded the time for the first 20 iterations. We used five trial solutions and a discrete distribution made of twelve realizations. During the course of the 20 iterations 66,320 linear programs have been solved. To make runs comparable we worked with a random but fixed sampling (line 12 of the algorithm) in all implementations. All experiments were carried out on a PC with an Intel i7-680 chip running Windows 7 (64bit) with GAMS version 23.7.0 and Cplex 12.2.0.2. We implemented three versions of the algorithm:

Traditional: This is a GAMS model implementing the SDDP algorithm with traditional GAMS programming flow control structures like loop. This traditional version has been tested with three different ways of calling the LP solver which is parameterized by the GAMS option solvelink. ChainScript, which is the default in GAMS, produces for each SOLVE statement some scratch files on disk containing the model instance, it also dumps the entire GAMS database into a scratch file and stops the GAMS runtime system leaving all computer resources to the solver job. After the solver job terminates, the GAMS runtime system reinitializes itself from the GAMS database scratch file and continues with execution of the GAMS program. CallModule also creates creates files for a model instance but the GAMS runtime system stays in memory while the solver job runs. LoadLibrary communicates the model instance through memory and initiates the solver through a shared library. This implementation is available at http://www.gams.com/modlib/adddocs/sddp_trad.gms.

GUSS: This implementation replaces parts of the traditional loop constructs in the traditional GAMS model by scenario based SOLVE statements using GUSS as discussed above. This model is part of the GAMS Model Library (http://www.gams.com/modlib/libhtml/sddp.htm)

Concert: This implementation is based on ILOG Concert Technology, a programming interface to generate and solve linear, quadratic and constraint programming based models with solvers available from the IBM Cplex Optimization Studio. This particular C++ implementation used Concert to generate linear programming model and solve them with Cplex. The $\mathrm{C}++$ program is available at http://www.gams.com/modlib/ adddocs/sddp.cpp. The model data (and the random data for sampling) comes from GAMS through the Gams Data eXchange (GDX).

All three implementations build on the same core LP technology, the Cplex dual simplex engine. The accumulated time spent in the core Cplex optimizer (CPXlpopt) for the 66,320 linear programs amounts to approximately $110 \mathrm{sec}-$ 
onds and differs by less than $1.5 \%$ between the different implementations. The following table gives the total running times of the different implementations:

\begin{tabular}{|l|r|}
\hline Traditional with \%Solvelink. ChainScript\% & $7204 \mathrm{sec}$ \\
Traditional with \%Solvelink. CallModule\% & $2481 \mathrm{sec}$ \\
Traditional with \%Solvelink.LoadLibrary\% & $1221 \mathrm{sec}$ \\
GUSS & $392 \mathrm{sec}$ \\
\hline ILOG Concert Technology & $210 \mathrm{sec}$ \\
\hline
\end{tabular}

As expected the traditional model becomes faster with a tighter solver communication. The GUSS implementation improves the running time by a factor of more than three compared to the fastest traditional run. The table also shows that the GUSS implementation is slower by a factor of less than two compared to the Concert implementation.

GAMS and other algebraic modeling system are widely accepted as rapid prototyping environments for models and algorithms that require the solution of mathematical optimization problems. Opinions diverge when it comes to selecting the software for deployment of such models and algorithms. One of the most frequent arguments for reimplementing a model in a compiled language with a native solver interface has been the running time overhead of an interpreted language like GAMS. The experiments of this section quantify this overhead (at least for this particular algorithm) and improve the basis for a cost-benefit analysis for the different deployment options.

\subsection{Conclusion}

While modeling systems such as GAMS, AMPL and AIMMS are often used to prototype and solve optimization problems from large classes of application domains, they have typically been slow at solving collections of models that have simple data changes of a core model. Specific instances that exhibit these difficulties have been described above, ranging from data envelopment analysis, classifier validation, and decomposition approaches such as SDDP, Lagrangian relaxation and Benders approach [4, 7, 11, 14].

We have demonstrated a simple language extension, termed GUSS, that facilitates the gathering of data from different sources/symbols that define the collection of models (that we term scenarios), the procedure that updates a base model instance with this scenario data and then solves this updated model instance, and the mechanism to scatter the scenario results to symbols in the GAMS database. This extension is very easy to incorporate into existing models, and the methodology we use to communicate the information to the solver engine is generalizable to more complex data updating schemes.

The extension can be used with any existing model type within the GAMS environment, and allows data parameters that are present in an existing 
model to be identified (and updated) using a scenario index. We have demonstrated its utility on a number of example applications and have shown distinct improvements in speed of processing these collections of models. We believe that models updated to use GUSS will be competitive with native implementations of decomposition algorithms, but will have the distinct advantage that they will be much easier to code, available to a modeler to tailor to a specific idea, and enable new suites of problems to be solved directly within the modeling system.

\section{References}

1. Banker, R.D., Charnes, A., Cooper, W.W.: Some models for estimating technical and scale inefficiencies in Data Envelopment Analysis. Management Science 30(9), 1078$1092(1984)$

2. Banker, R.D., Morey, R.C.: Efficiency analysis for exogenously fixed inputs and outputs. Operations Research 34(4), 513-521 (1986)

3. Banker, R.D., Morey, R.C.: The use of categorical variables in Data Envelopment Analysis. Management Science 32(12), 1613-1627 (1986)

4. Benders, J.F.: Partitioning procedures for solving mixed-variables programming problems. Numerische Mathematik 4(1), 238-252 (1962)

5. Birge, J.R., Louveaux, F.: Introduction to Stochastic Programming. Springer Verlag (1997)

6. Bussieck, M.R., Ferris, M.C., Meeraus, A.: Grid enabled optimization with GAMS. INFORMS Journal on Computing 21(3), 349-362 (2009)

7. Carøe, C.C., Schultz, R.: Dual decomposition in stochastic integer programming. Operations Research Letters 24, 37-45 (1999)

8. Charnes, A., Cooper, W., Lewin, A.Y., Seiford, L.M.: Data Envelopment Analysis: Theory, Methodology and Applications. Kluwer Academic Publishers, Boston, MA (1994)

9. Charnes, A., Cooper, W.W., Rhodes, E.: Measuring the efficiency of decision making units. European Journal of Operational Research 2, 429-444 (1978)

10. Cooper, W.W., Seiford, L.M., Tone, K.: Data Envelopment Analysis: A Comprehensive Text with Models, Applications, References and DEA-Solver Software. Kluwer Academic Publishers, Boston, MA (2000)

11. Dantzig, G.B., Wolfe, P.: Decomposition principle for linear programs. Operations Research 8, 101-111 (1960)

12. Efron, B., Tibshirani, R.: Improvements on cross-validation: The $.632+$ bootstrap method. Journal of the American Statistical Association 92, 548-560 (1997)

13. Farrell, M.J.: The measurement of productive efficiency. Journal of the Royal Statistical Society, Series A (General) 120(3), 253-290 (1957)

14. Ferris, M.C., Maravelias, C.T., Sundaramoorthy, A.: Simultaneous batching and scheduling using dynamic decomposition on a grid. INFORMS Journal on Computing 21(3), 398-410 (2009)

15. Ferris, M.C., Voelker, M.M.: Slice models in general purpose modeling systems: An application to DEA. Optimization Methods and Software 17, 1009-1032 (2002)

16. Geisser, S.: Predictive Inference. Chapman and Hall, New York (1993)

17. Kohavi, R.: A study of cross-validation and bootstrap for accuracy estimation and model selection. In: Proceedings of the Fourteenth International Joint Conference on Artificial Intelligence 2, p. 11371143. Morgan Kaufmann, San Mateo (1995) 
18. Olesen, O.B., Petersen, N.C.: A presentation of GAMS for DEA. Computers and Operations Research 23(4), 323-339 (1996)

19. Pereira, M.V.F., Pinto, L.M.V.G.: Stochastic optimization of a multireservoir hydroelectric system: A decomposition approach. Water Resources Research 21(6), 779-792 (1985)

20. Pereira, M.V.F., Pinto, L.M.V.G.: Multi-stage stochastic optimization applied to energy planning. Mathematical Programming 52, 359-375 (1991)

21. Picard, R., Cook, D.: Cross-validation of regression models. Journal of the American Statistical Association 79, 575-583 (1984)

22. Seiford, L.M., Zhu, J.: Sensitivity analysis of DEA models for simultaneous changes in all the data. Journal of the Operational Research Society 49, 1060-1071 (1998)

23. Simar, L., Wilson, P.W.: Sensitivity analysis of efficiency scores: How to bootstrap in nonparametric frontier models. Management Science 44(1), 49-61 (1998)

24. Thanassoulis, E., Boussofiane, A., Dyson, R.G.: Exploring output quality targets in the provision of perinatal care in England using DEA. European Journal of Operations Research 60, 588-608 (1995)

25. Velaśquez, J., Restrepo, P., Campo, R.: Dual dynamic programming: A note on implementation. Water Resources Research 35(7) (1999) 